\title{
Assessment of using hollow fibre microfiltration in treating lake water
}

\begin{abstract}
The main objective of this study was to determine the reliability of using microfiltration system which is composed of a coagulant such as ferric chloride (pretreatment substance) and hollow fibre membrane as filtering medium for improving water quality. In this study, raw water samples were collected from a lake located at the Faculty of Engineering, Universiti Putra Malaysia. The experiments were carried out in three stages: the first stage used distilled water, the second stage used lake water stored in a tank and the third stage used lake water stored in the tank and mixed with ferric chloride as coagulant. A storage tank and a separation tank were used; the storage tank is used to store water pumped from the lake (used in the second stage) while in the third stage, the stored water in tank was used for pretreatment or coagulation with optimum dosage of ferric chloride (determined from jar test). The experimental results show that the flux obtained from second and third stages was low. For lake water stage, the best flux was found to be $4.97 \mathrm{l} / \mathrm{m} 2 / \mathrm{h}$. The distilled water stage shows that shaft rotational speed of peristaltic pump was one of the factors affecting the flux. Therefore, a pump shaft rotational speed of $7 \mathrm{rpm}$ was chosen based on flux and suction pressure. The experimental results show the ability of microfiltration using hollow fibre membrane together with ferric chloride as coagulant to produce treated water with $97 \%$ turbidity removal and $85 \%$ removal of total suspended solids (TSS). For treated raw water, it is found that the removals of TSS were ranged from 71.7 to $91.6 \%$ when alum was used as a coagulant while the TSS removals were ranged from 74.4 to $89.20 \%$ when ferric chloride was used as a coagulant. In this study, it is found that the optimum dosages of ferric chloride and alum were 4 and $20 \mathrm{mg} / \mathrm{l}$, respectively. Although the percentage removal of TSS is slightly higher when alum is used as a coagulant compared with using ferric chloride, it is recommended to use ferric chloride as a coagulant due to its economical and health advantages. Furthermore, ferric chloride can be used in wide range of $\mathrm{pH}$ values while alum can only be effective when $\mathrm{pH}$ value around 7 .
\end{abstract}

Keyword: Lake water; Treatment; Coagulation; Microfiltration 